\title{
Adsorption of polyiodobenzene molecules on the Pt(111) surface using van der Waals density functional theory.
}

\author{
Karen Johnston ${ }^{1}$, Rengin Peköz ${ }^{2}$ and Davide Donadio ${ }^{2,3,4,5}$ \\ ${ }^{1}$ Department of Chemical and Process Engineering, University of \\ Strathclyde, 75 Montrose Street, Glasgow G1 1XJ, United Kingdom \\ ${ }^{2}$ Max Planck Institute for Polymer Research, Ackermannweg 10, \\ Mainz 55128, Germany \\ ${ }^{3}$ Donostia International Physics Center, Paseo Manuel de Lardizabal, \\ 4, E-20018 Donostia-San Sebastian, Spain \\ ${ }^{4}$ IKERBASQUE, Basque Foundation for Science, Alameda Urquijo \\ 36-5, 48011 Bilbao, Spain \\ ${ }^{5}$ Department of Chemistry, University of California Davis, One \\ Shields Avenue, Davis, CA, 95616, U.S.A.
}

September 14, 2015

\begin{abstract}
Adsorption of aromatic molecules on surfaces is widely studied due to applications in molecular electronics. In this work, the adsorption of iodobenzene molecules on the Pt(111) surface has been studied using density functional theory. Iodobenzene molecules, with various numbers of iodine atoms, have two non-dissociative adsorption minima. One structure exhibits chemisorption between the ring and the surface (short-range) and the other structure exhibits chemisorption between the iodine ions and the surface (long-range). Both structures have a strong van der Waals interaction with the surface. In general, the adsorption energy increases as the number of iodine atoms increases. The dissociated structure of monoiodobenzene was investigated, and the dissociation barrier and the barrier between the short- and longrange states were compared.
\end{abstract}




\section{Introduction}

The adsorption of aromatic molecules on surfaces is a topic that has received a lot of attention due to their potential use in organic electronics and nanolithography devices [1, 2, 3]. Functionalised aromatic rings are also known to self-assemble on metal surfaces, forming a variety of structures, including wire-like structures [4] and branched structures [5]. In addition, functionalized aromatic molecules can exhibit a bistability on metal surfaces $[6,7]$ paving the way for molecular switching applications. However, functionalised aromatic rings are also considered pollutants, and catalytic routes using platinum for breaking down such molecules are being developed [8].

The adsorption of iodobenzene on metal surfaces has been intensively studied, due to the ease of iodine removal in coupling reactions, such as Sonogashira coupling $[9,10,11]$. On $\mathrm{Au}(111)$ iodobenzene is mainly reversibly adsorbed although partial decomposition into iodine and adsorbed biphenyl on the surface was also observed [12]. On $\mathrm{Cu}(111)$, iodobenzene was found to undergo $\mathrm{C}-\mathrm{I}$ dissociation with the phenyl rings adsorbing flat on the surface [13]. However, an STM study did observe non-dissociated structures of iodobenzene on $\mathrm{Cu}(111)$ [14].

Adsorption on the $\operatorname{Pt}(111)$ surface is of particular interest due to a bistability of two non-dissociated states exhibited by halogenated aromatic molecules [7] leading to the possibility of tuning the adsorption structure. An experimental study of iodobenzene on $\mathrm{Pt}(111)$ observed non-dissociative adsorption of monoiodobenzene (MIB) below $175 \mathrm{~K}$ [15] and above this temperature they observed dissociative adsorption with $\mathrm{C}-\mathrm{I}$ bond scission.

In this work we focus on the adsorption of iodobenzene on the $\mathrm{Pt}(111)$ surface and investigate the dissociative and non-dissociative adsorption structures and energetics using density functional theory with van der Waals $(\mathrm{vdW})$ interactions. The structure and energetics of these states, and the dissociation barrier for MIB, have been investigated and compared to experimental results.

\section{Method}

Calculations were performed with the VASP 4.6 code, which implements density functional theory with a plane wave basis set $[16,17]$. The cutoff energy for the valence electrons was $400 \mathrm{eV}$ and the core electrons were described using the projector augmented wave method $[18,19]$. Both a standard generalised gradient approximation (GGA), namely PBE [20, 21], and 
vdW-DF [22] exchange and correlation functionals were used to investigate the chemical bonding and vdW interactions. For the vdW-DF calculations the exchange energy was described using the PBE functional, since it was shown to give good adsorption energies for our previous work on halobenzenes on platinum [7], benzene on gold [23] and on silicon [24]. The calculated lattice constant for platinum using vdW-DF with $\mathrm{PBE}$ exchange is $4.03 \AA$, which is about $3 \%$ larger that the experimental value of $3.92 \AA$ [25] and is typical for the vdW-DF functional [26]. The PBE lattice constant is $3.98 \AA$.

The surface was set up using four atomic layers with 16 atoms per layer, which corresponds to a coverage of $0.41 \mathrm{ML}$, using the definition of Ihm et al. [27]. A larger cell of 30 atoms per layer, corresponding to a coverage of $0.22 \mathrm{ML}$ was also considered. Cabibil et al. estimated the saturation coverage of MIB to be one molecule for every $10 \mathrm{Pt}$ atoms [15], which is slightly higher than 0.41 ML. The bottom two layers of the Pt surface were held fixed during the ionic relaxations and the relaxations were stopped when the maximum force on the atoms was less than $10 \mathrm{meV} / \AA$.

The convergence with respect to the slab thickness was tested by increasing the number of atomic layers from 4 to 6 for several of the the $0.41 \mathrm{ML}$, short-range iodobenzene adsorption structures, and the bottom four atomic layers were fixed. This increase in slab thickness results in a stronger adsorption energy of $\approx 0.2 \mathrm{eV}$ for all adsorbed iodobenzene molecules but does not change the trend, so we chose to use 4 atomic layers in all subsequent calculations.

The height of the supercell is $37 \AA$ in all cases, giving a large vacuum region of around $26 \AA$, which minimises any effect due to the artificial dipole moment across the cell. This was checked by increasing the vacuum region by $10 \AA$ and by applying a dipole correction and in both cases the energy changed by less than $0.02 \mathrm{eV}$. The dimensions of all the cells used for the $\mathrm{PBE}$ and vdW-DF functionals are summarised in Table 1.

Table 1: Cell dimensions for different functionals and coverages.

\begin{tabular}{|l|r|rrr|}
\hline Functional & Coverage (ML) & $\mathrm{a}(\AA)$ & $\mathrm{b}(\AA)$ & $\mathrm{c}(\AA)$ \\
\hline PBE & 0.41 & 11.2515 & 9.744 & 37.0 \\
PBE & 0.22 & 14.065 & 14.616 & 37.0 \\
vdW-DF & 0.41 & 11.404 & 9.876 & 37.0 \\
vdW-DF & 0.22 & 14.255 & 14.814 & 37.0 \\
\hline
\end{tabular}

We note that, in several cases, molecules with multiple iodine atoms 
spontaneously dissociated. Therefore, to find a stable non-dissociated structure, we used starting configurations based on the stable structures of similar molecules.

The nudged elastic band calculation was performed using the implementation in the VASP package $[28,29]$ and images were relaxed to $50 \mathrm{meV} / \AA$.

\section{Results and Discussion}

\subsection{Adsorption Site}

Benzene is known to be stable on the bridge site on $\mathrm{Pt}(111)$ with an orientation of $30^{\circ}$ (denoted B30) with the crystal axis [30, 31, 32, 33]. For benzene this is followed by the hep hollow site and fcc hollow sites, both with a $0^{\circ}$ angle [33] (denoted FCC0 and HCP0, respectively). Other halogenated benzenes, such as difluorobenzene and hexafluorobenzene were also found to be most stable on the B30 site (see Supporting Information in Ref. [7]).

To check that the large iodine atoms do not affect the energetic ordering of the adsorption sites significantly, different orientations of $p$-diiodobenzene (DIB) in a chemisorbed configuration were considered. The B30 configuration was found to be the most stable with an adsorption energy of $-2.03 \mathrm{eV}$. The only other metastable configurations were HCP0 and FCC0, with adsorption energies of $-1.73 \mathrm{eV}$ and $-1.65 \mathrm{eV}$, respectively. DIB on the other sites proved to be unstable in a ring-bonded state and spontaneously moved to an iodine-bonded state. When the DIB was placed on the HCP30 site it moved back towards the bridge site. Therefore, for the following investigations we have focused on the B30 configuration.

\subsection{Bistability}

To investigate the interaction of DIB with the surface we calculated the adsorption energy curve using both PBE and vdW-DF functionals for a coverage of $0.41 \mathrm{ML}$. The adsorption energy curves were calculated by placing the DIB ring on the surface with B30 configuration and keeping the $x y z$ coordinates of two opposite $\mathrm{C}$ atoms in the ring fixed. The distance is defined as the distance from the fixed carbon atoms in the molecule to the position of the top layer of the relaxed, isolated Pt slab.

It can be seen from Fig. 1 that DIB exhibits a bistability in the adsorption energy curve with both types of functionals having a double well. The $\mathrm{PBE}$ functional gives a minimum of $-0.64 \mathrm{eV}$ at $3.2 \AA$ and a deeper minimum of $-1.14 \mathrm{eV}$ at $2.1 \AA$. The states corresponding to these minima are 
similar to the chemi- and physisorbed states observed for other halogenated benzene [7] but they will be denoted as short-range (SR) and long-range (LR) states, since both states involve significant electronic displacement, which is discussed later.

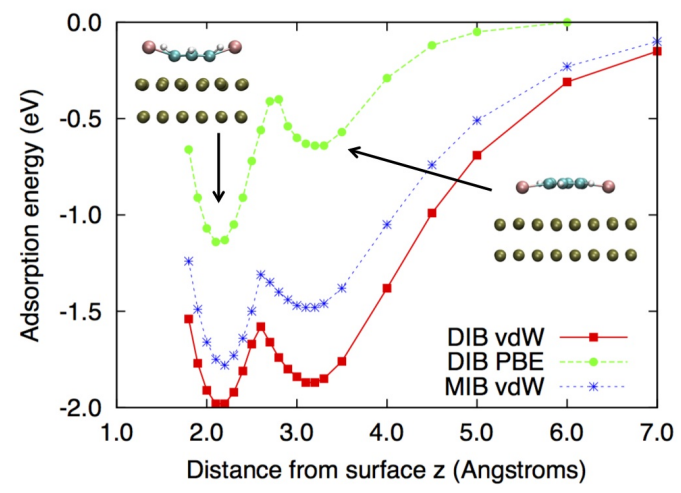

Figure 1: The adsorption energy curve for MIB and DIB on $\operatorname{Pt}(111)$ with a coverage of $0.41 \mathrm{ML}$. The short- and long-range structures of DIB are shown as insets.

The inclusion of vdW interactions has a strong effect on the adsorption energy curve of DIB and strengthens the adsorption energy of the shortrange minimum from -1.14 to $-1.98 \mathrm{eV}$. In addition, the bistability becomes more pronounced since the relative depth of the long-range minimum is increased with respect to the short-range minimum so that both states have closer adsorption energies.

Bistability was observed for other dihalobenzene molecules $[6,7]$. However, DIB displays a marked difference in adsorption behaviour. First, DIB has a much stronger adsorption on $\mathrm{Pt}(111)$ than other dihalobenzene molecules. For $0.41 \mathrm{ML}$ coverage, the long range vdW-DF minimum at $3.2 \AA$ is around $-1.87 \mathrm{eV}$. This is much deeper than for other dihalobenzenes, which have weaker long-range minima ranging from $-0.97 \mathrm{eV}$ for difluorobenzene to $-1.36 \mathrm{eV}$ for dibromobenzene [7]. For the other dihalobenzene molecules the long-range minimum was almost entirely due to vdW forces, whereas for DIB the minimum has a strong chemical nature due to the interaction between the platinum and iodine ions. The short-range minimum of $-1.97 \mathrm{eV}$ is significantly deeper than the short-range minima of other halobenzene molecules, which range from -1.31 to $-1.55 \mathrm{eV}$ for difluoroto dibromobenzene [7]. In all subsequent sections, only the vdW adsorption 
energies will be presented.

The adsorption energy curve for MIB using the vdW functional also shows a double well with the positions of the minima being similar to DIB, as shown in Fig. 1. The short-range minimum for MIB is $0.20 \mathrm{eV}$ shallower than that of DIB, whereas the long-range minimum is shallower by $0.39 \mathrm{eV}$. This reflects the different bonding types in the two minima, which is discussed later.

We note that the constraint on the carbon atoms of the molecule results in a slightly higher adsorption energy compared to that of the fully optimised geometry, and therefore Fig. 1 represents an upper limit to the PES. For DIB, calculated with vdW, the short-range minimum is $-1.98 \mathrm{eV}$, and the fully optimised value (see Table 2 ) is $-2.03 \mathrm{eV}$. For the long range minimum the adsorption energy is $-1.87 \mathrm{eV}$, and the fully optimised value is $-1.97 \mathrm{eV}$. For MIB with vdW, the adsorption energies are $-1.78 \mathrm{eV}$ (constrained) and $-1.81 \mathrm{eV}$ (relaxed) for the short-range structure and $-1.48 \mathrm{eV}$ (constrained) and $-1.55 \mathrm{eV}$ (relaxed) for the long-range structure.

\subsection{Variation of adsorption energy minima}

The effect on the two adsorption minima of increasing the number of iodine atoms was investigated using the vdW-DF functional. The adsorption energies for the short-range (SR) and long-range (LR) structures, for both $0.22 \mathrm{ML}$ and 0.41 ML coverage, are shown in Fig. 2.

For benzene, MIB and DIB, the short range structures are energetically lower than the long range ones. However, the short- and long-range minima for molecules with three or more iodine atoms are very similar. This behaviour is different than other halogenated molecules previously studied [7], where a clear crossover from short-range to long-range stability was found. In addition, the adsorption energies of the iodobenzene molecules in the short- and long-range structures, are much lower than the analogous structures of benzene and the other halogenated benzene molecules.

In general, the short-range and long-range minima deepen as the number of iodine atoms is increased. For low coverage, the short-range minima decrease approximately linearly with increasing number of iodine atoms. For high coverage the minima also decrease approximately linearly, however, the short-range structure of hexaiodobenzene (HIB) at high coverage has an anomalously high adsorption energy. This anomalous behaviour is due to a repulsion between the molecule and its periodic images. This effect is also seen for certain rotations of the other molecules as described below.

To investigate the effect of rotation on the repulsion between nearest 


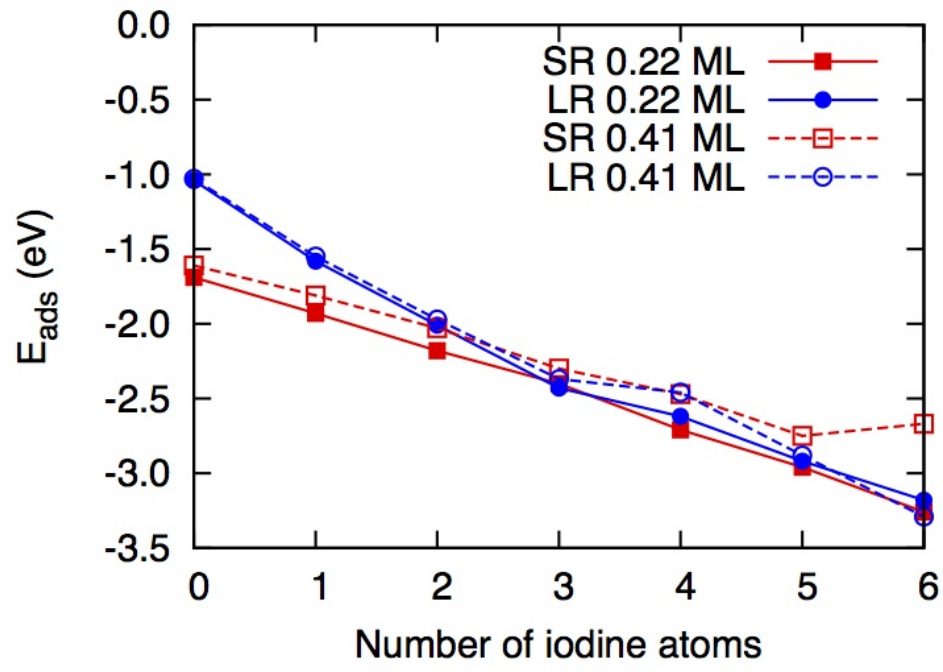

Figure 2: vdW adsorption energies of short-range (SR) and long-range (LR) minima of polyiodobenzene molecules. Solid symbols correspond to a coverage of $0.22 \mathrm{ML}$ and hollow symbols to the higher coverage of $0.41 \mathrm{ML}$. For each case the most favorable adsorption energy is shown.

neighbours, we calculated the adsorption energies of different rotations of triiodobenzene (TriIB), tetraiodobenzene (TetIB) and pentaiodobenzene (PIB) molecules on the surfaces. The three possible rotations for TetIB and an explanation of the notation are shown in Fig. 3.

Fig. 2 shows only the lowest adsorption energies but adsorption energies for all rotations are presented in Table 2. The depths of the short-range minima for the different rotations vary by $\approx 0.3 \mathrm{eV}$ for TetIB and $\approx 0.4 \mathrm{eV}$ for PIB. For lower coverage these ranges are reduced to less than $0.2 \mathrm{eV}$ for both TetIB and PIB. For HIB, it is not possible to avoid the I-I repulsion between neighbours by rotating the molecule. For HIB's short-range structure, the difference in energy between the low and high coverages is $\approx 0.6 \mathrm{eV}$.

For the long-range minimum this repulsion is much weaker and the adsorption energies are similar for both coverages, with the higher coverage having a slightly higher energy. This is due to the fact that the long-range structures have more freedom to move on the surface. 


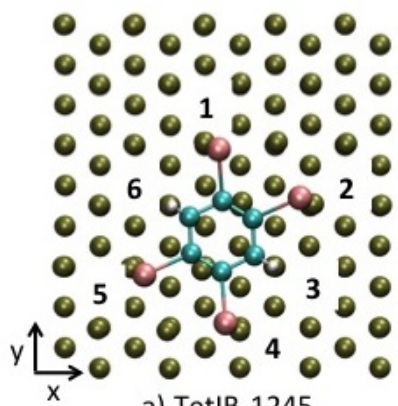

a) TetIB-1245

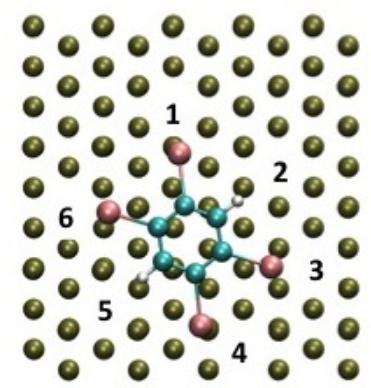

b) TetIB-1346

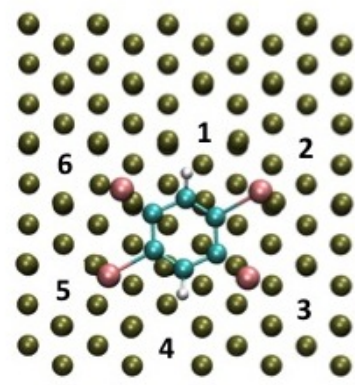

c) TetIB-2356

Figure 3: The three different rotation structures for TetIB in the short-range structure with $0.22 \mathrm{ML}$ coverage. The rotation is denoted by the position of carbon atoms, which are bonded to iodine atoms. The angles in Table 3 are defined as the angle between the axis through carbon atoms 2 and 5 and the $\mathrm{x}$-axis.

\subsection{Adsorption Geometries}

Geometries of the molecules in the short-range and long-range minima are shown in Fig. 4 for $0.22 \mathrm{ML}$ coverage. The main difference between these structures is that the short-range structures are mainly ring-bonded, with the ring around $2.2 \AA$ from the surface, whereas the long-range structures are mainly bonded via the iodine atoms with the ring at a distance of $3.1 \AA$ from the surface. All geometries shown are for the vdW functional, however, adsorption geometries using the PBE and vdW-DF functionals are almost indistinguishable.

\subsubsection{Short-range structures}

The short-range geometries have the ring positioned at around $2.2 \AA$ from the surface and the iodine atoms are bent upwards away from the surface. All the molecules are stable on the $\mathrm{B} 30$ site. The $\mathrm{C}-\mathrm{I}$ and $\mathrm{C}-\mathrm{H}$ bonds make a range of angles with the surface plane and, particularly for those with several iodine atoms, the angles can be quite high. The angles are shown in Fig. 5.

It is clear from Fig. 5 that, for the low coverage structure, the C-I and $\mathrm{C}-\mathrm{H}$ angles strongly depend upon the position on the surface. Positions 3 and 4 correspond to those where the $\mathrm{C}-\mathrm{I}$ or $\mathrm{C}-\mathrm{H}$ bond lies approximately above a Pt atom (top site), which pushes the bond upwards. The bonds in 
Table 2: Adsorption energies in $\mathrm{eV}, E_{\text {ads }}$, and charge displacement in units of electrons, $Q_{\text {disp }}$, for molecules of various rotations at 0.41 and $0.22 \mathrm{ML}$ coverages and for short-range (SR) and long-range (LR) structures. All data were calculated with the vdW functional.

\begin{tabular}{|c|c|c|c|c|c|c|c|c|}
\hline \multirow{3}{*}{$\begin{array}{l}\text { Molecule \& } \\
\text { rotation }\end{array}$} & \multicolumn{4}{|c|}{$0.41 \mathrm{ML}$} & \multicolumn{4}{|c|}{$0.22 \mathrm{ML}$} \\
\hline & \multicolumn{2}{|c|}{ SR } & \multicolumn{2}{|c|}{ LR } & \multicolumn{2}{|c|}{ SR } & \multicolumn{2}{|c|}{ LR } \\
\hline & $E_{\text {ads }}$ & $Q_{\text {disp }}$ & $E_{\text {ads }}$ & $Q_{\text {disp }}$ & $E_{\text {ads }}$ & $Q_{\text {disp }}$ & $E_{\text {ads }}$ & $Q_{\text {disp }}$ \\
\hline Benzene & -1.61 & 3.600 & -1.03 & & -1.69 & 3.854 & -1.04 & 0.947 \\
\hline & 81 & 4.158 & -1.55 & 1.4 & -1.93 & 4.562 & -1.58 & 1.650 \\
\hline & & 4.6 & -1.97 & & & 5.204 & -2.01 & \\
\hline Tri & & 4.680 & -2.37 & & & & & \\
\hline Tri & & 4.677 & -2.37 & & -2.40 & 5.279 & -2.43 & \\
\hline & .30 & 5.277 & -2.38 & & -2.71 & 6.359 & -2.62 & 281 \\
\hline & -2.1 & 4.423 & -2.23 & & -2. & 5.538 & -2.62 & 3.881 \\
\hline & .47 & 4.986 & -2.46 & 3.409 & -2.55 & 5.526 & -2.51 & 3.862 \\
\hline & -2.50 & 5.637 & -2.82 & & -2.90 & 6.802 & -2.91 & 3.460 \\
\hline & -2.41 & 5.978 & -2.83 & & -2.83 & 6.341 & -2.82 & 3.502 \\
\hline & -2.75 & 5.794 & -2.87 & 3.827 & -2.86 & 6.365 & -2.92 & 3.234 \\
\hline & -2.55 & 5.674 & -2.94 & & -2.96 & 6.816 & -2.78 & 3.025 \\
\hline & -2.38 & 5.221 & -2.88 & & -2.86 & 6.371 & -2.89 & 3.417 \\
\hline & -2.74 & 5.724 & -2.87 & 3.8 & -2.83 & 6.345 & -2.77 & 2.967 \\
\hline HexIB & -2.67 & 5.987 & -3.29 & 4.258 & -3.26 & 7.714 & -3.18 & 3.596 \\
\hline
\end{tabular}

these positions show a trend as the number of iodine molecules is increased. First we consider those molecules with a $\mathrm{C}-\mathrm{H}$ bond in this location, namely, MIB, DIB, TetIB-1245 and PenIB-12345. For these molecules the bond angle increases as the number of iodine atoms is increased. For the $\mathrm{C}-\mathrm{I}$ bond, the angles are generally higher than for the $\mathrm{C}-\mathrm{H}$ bond, and also approximately increase as the number of iodine atoms increases. The positions 1, 2, 4 and 5 show the opposite trend. Here, the angle decreases slightly as the number of iodine atoms is increased, with HIB having the smallest angles in all positions.

For the high coverage of $0.41 \mathrm{ML}$, the trend is not so clear. The angles for several structures are higher than for the 0.22 ML coverage, which is likely to be due to the repulsion between the molecule's periodic images. In particular, HIB, PenIB-13456, TetIB-1245 and TetIB-1346 show a high angle for the iodine in position 4 . The iodines in this position are closest 

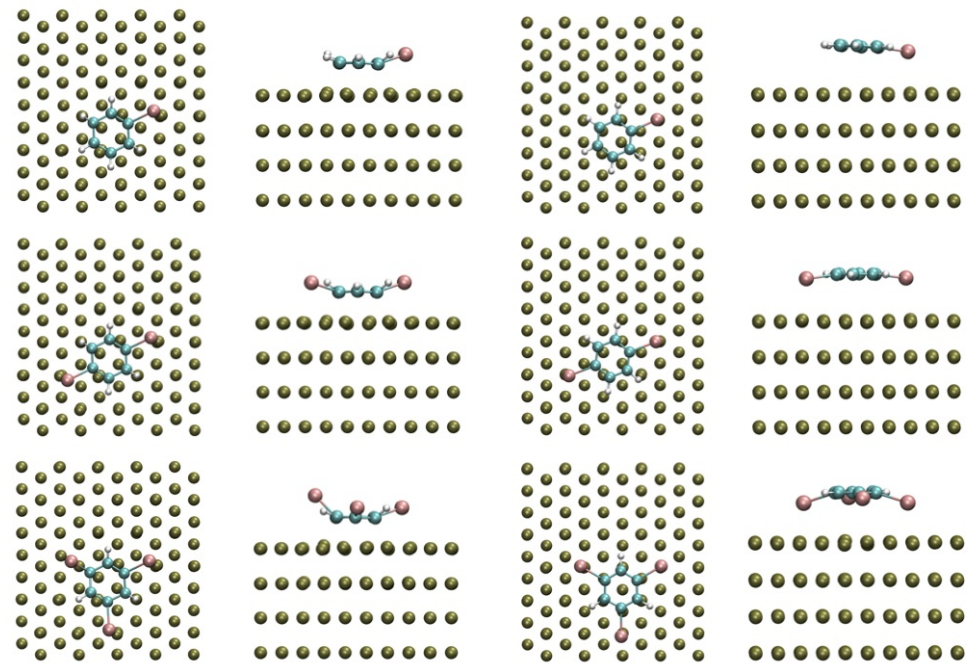

0000

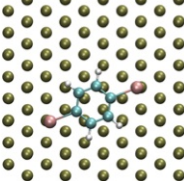

- ०००००००००

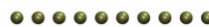
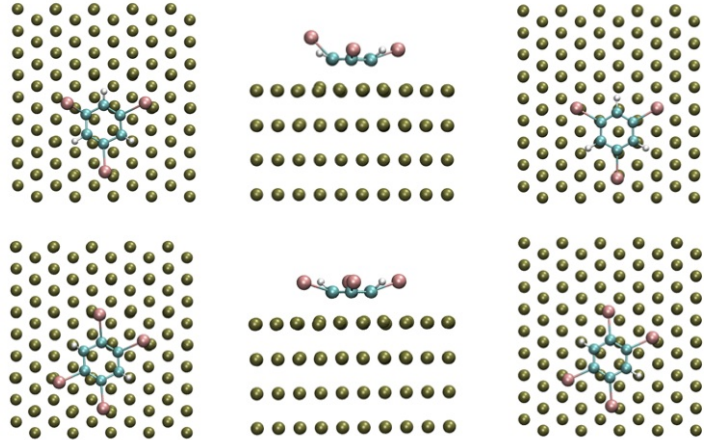

000000000

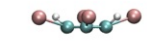

0000000000

००००००००००

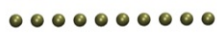

००००००००००
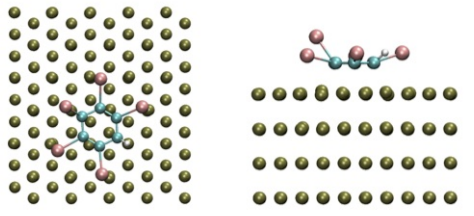

$\because \because 0000$

$\because \because \div \div:$

$\because 00: 0$

$\because 08:-$

$\because: 00: 0$

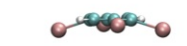

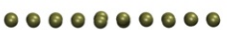

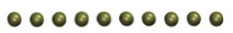

००००००००००

๑०००००००००
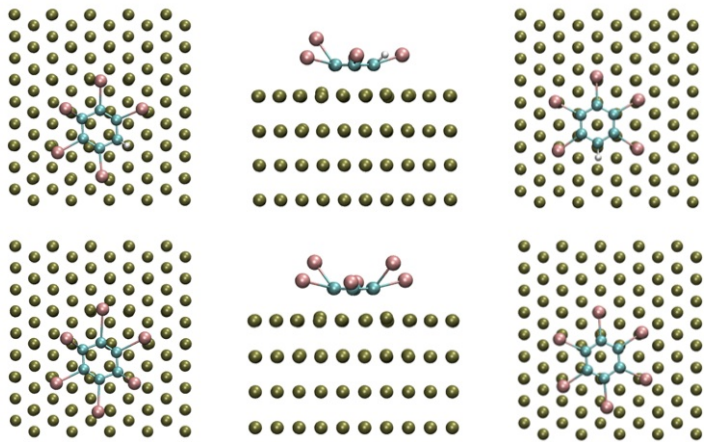

araro

0000000000

०००००00000

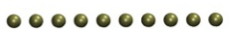

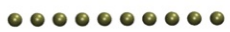

0-000

- ०००००००००

०००००००000

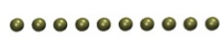

००००००००००

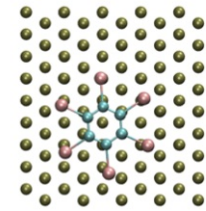

80000-8

- 000000000

๑००००००००८

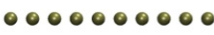

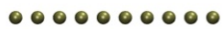

Figure 4: Adsorption geometries of energetically favorable short-range (left two columns) and long-range (right two columns) structures for polyiodobenzene molecules with $0.22 \mathrm{ML}$ coverage. The number of iodine atoms increases from top to bottom.

to the iodine in position 1 of the periodic image, which has a lower angle of between $10^{\circ}$ and $15^{\circ}$. Conversely for PIB-12345, the angle at position 1 

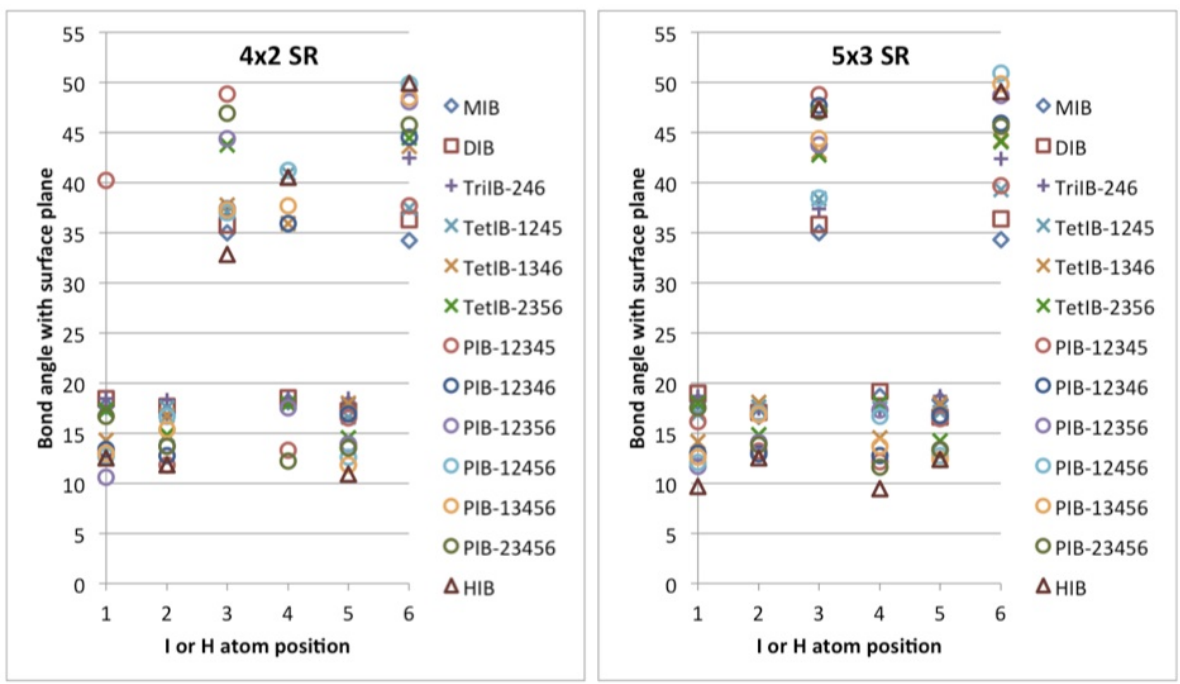

Figure 5: The distribution of angles that the $\mathrm{C}-\mathrm{H}$ and $\mathrm{C}-\mathrm{I}$ bonds make with the surface plane for the short-range structures at 0.41 ML (left), and $0.22 \mathrm{ML}$ coverage (right).

is high and the angle at position 4 remains low. These structures with the high angles correspond to higher energy rotations and are higher in energy than the corresponding structures for low coverage.

\subsubsection{Long-range structures}

For the long range structure the ring is not strongly interacting with the surface and the molecule is not always located at the B30 site. Molecules with few iodine atoms are stable on the bridge site, however, as the number of iodine atoms is increased the molecule tends to prefer to be on an HCP or FCC site. Some molecules are not centred above any high-symmetry site and are referred to as unclassified. The adsorption sites and angles for all structures are given in Table 3.

For MIB at low coverage, the ring remains approximately centred on the bridge site. The iodine atom is above a top site and the molecule is slightly tilted with respect to the surface plane, which is due to the interaction between the iodine atom and the surface. DIB remains ring-centred on the bridge site, with the iodine atoms close to, but not exactly on, the top site. For TriIB-246, the entire molecule has slightly shifted so that the ring is 
Table 3: Adsorption geometries of iodobenzene molecules of various rotations in the long- and short-range structure at $0.22 \mathrm{ML}$ using the vdW functional. The letter shows which site the ring is centred above: top $(\mathrm{T})$, fcc $(\mathrm{F})$, hcp $(\mathrm{H})$, bridge (B) or unclassified (U). The corresponding number is the angle between the axis through opposite $\mathrm{C}$ atoms at the 2 and 5 positions and the crystal $x$-axis, as shown and described in Fig. 3.

\begin{tabular}{|l|ll|lr|rr|rr|}
\hline Molecule \& & \multicolumn{4}{|c|}{0.41} & \multicolumn{4}{c|}{$0.22 \mathrm{ML}$} \\
rotation & \multicolumn{2}{|c|}{ SR } & \multicolumn{2}{c|}{ LR } & \multicolumn{2}{c|}{ SR } & \multicolumn{2}{c|}{ LR } \\
\hline Benzene & B & 30.0 & B & 29.9 & B & 30.0 & B & 29.9 \\
MIB & B & 29.5 & B & 24.0 & B & 29.5 & B & 23.9 \\
DIB & B & 29.1 & B & 20.9 & B & 28.8 & B & 21.4 \\
\hline TriIB-135 & B & 29.7 & F & 28.6 & - & - & - & - \\
TriIB-246 & B & 29.8 & H & 30.0 & B & 29.8 & H & 30.1 \\
\hline TetIB-1245 & B & 28.2 & B & 12.9 & B & 29.4 & B & 29.2 \\
TetIB-1346 & B & 27.4 & B & 12.1 & B & 31.4 & B & 37.8 \\
TetIB-2356 & B & 28.2 & B & 18.8 & B & 28.4 & B & 21.0 \\
\hline PenIB-12345 & B & 29.6 & B & 14.2 & B & 29.1 & U & 34.7 \\
PenIB-12346 & B & 26.4 & U & 10.1 & B & 29.7 & U & 41.8 \\
PenIB-12356 & B & 27.9 & B & 16.9 & B & 28.4 & F & 31.2 \\
PenIB-12456 & B & 28.4 & U & 11.0 & B & 29.2 & U & 35.0 \\
PenIB-13456 & B & 28.0 & U & 8.9 & B & 30.1 & F & 34.7 \\
PenIB-23456 & B & 29.0 & B & 18.5 & B & 28.5 & F & 35.2 \\
\hline HexIB & B & 28.8 & B & 13.3 & B & 27.4 & F & 34.7 \\
\hline
\end{tabular}

centred above a hollow site and all the iodine atoms are on top sites. All the TetIB molecules remain on the bridge site but the PenIB molecules move to different sites.

For high coverage, the energetically favorable sites are quite different to those at low coverage and there is a large variation in the angle of the $\mathrm{C}-$ $\mathrm{C}$ axis with the crystal axis. However, given the large number of possible configurations it is not feasible to check every possible site and rotation angle, and we cannot rule out the possibility that there may be lower energy configurations for some of the long-range structures.

For long-range structures, the ring is approximately $3.1 \AA$ from the surface and the iodine atoms are mainly bent down towards the surface. The $\mathrm{C}-\mathrm{I}$ and $\mathrm{C}-\mathrm{H}$ angles for the long-range structures are presented in Fig. 6 .

It is more difficult to discern a trend in the angles for the long range 


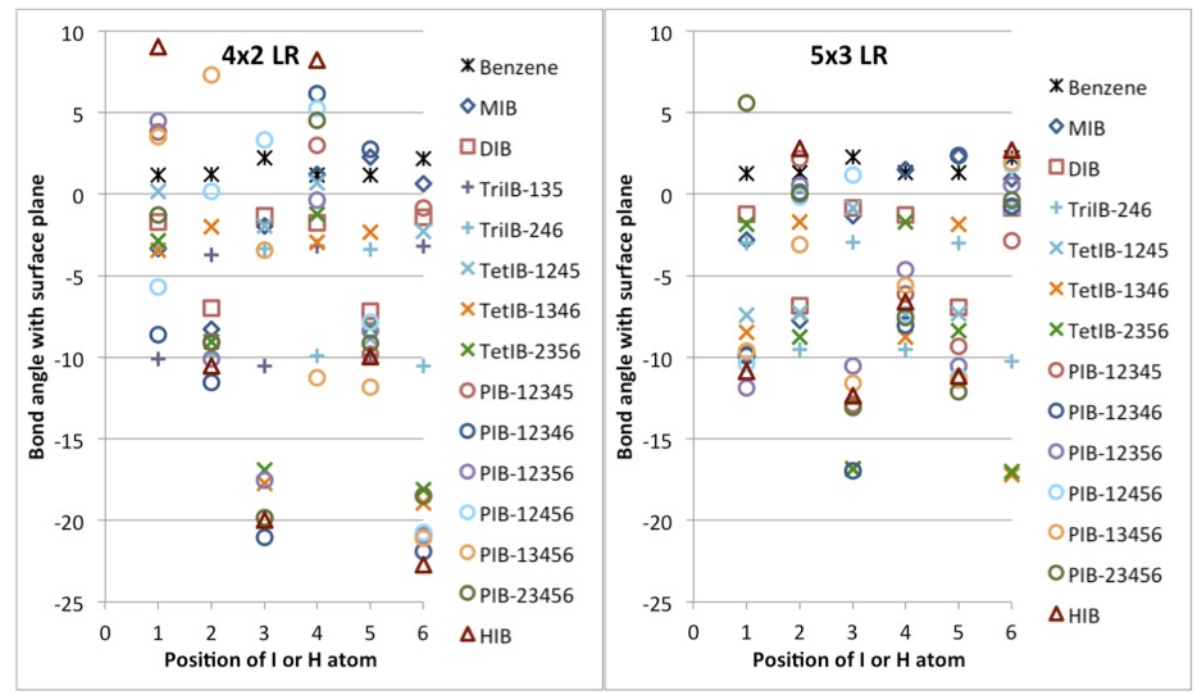

Figure 6: The distribution of angles that the $\mathrm{C}-\mathrm{H}$ and $\mathrm{C}-\mathrm{I}$ bonds make with the surface plane for the long-range structures at 0.41 ML (left), and $0.22 \mathrm{ML}$ coverage (right).

structures, even at low coverage. For benzene the variation in the angles is very small. For MIB, the iodine ion (position 2) is bent down towards the surface with an angle of $-8.3^{\circ}$. Similar behaviour is seen for the iodine ions in DIB and TriIB-246. However, this behaviour changes once there are four iodine ions on the molecule. This is likely to be due to fact that the molecules have different sites and angles and, hence, different interactions with the surface atoms. In general, the range of angles becomes larger as the number of iodine ions is increased.

Several long-range adsorption structures differ markedly between high and low coverages. For both coverages, benzene, MIB and DIB have similar angles. However, molecules with a large number of iodine atoms the spread of the angles increases markedly, in particular HIB has angles ranging from $-23^{\circ}$ to $9^{\circ}$. Overall, the angles are higher for the higher coverage system than for the lower coverage system, which again indicates a repulsion between molecules and their periodic images. 


\subsubsection{Tilted structure}

In addition to the long and short-range structures described above, we found a tilted structure for TetIB and PenIB for high coverage. In this configuration, the ring shows a slight distortion and tilt with respect to the surface. However, the tilted structures have a weaker adsorption energy than all long-range structures and several short-range structures, so these structures will not be further discussed.

\subsection{Charge density}

The difference in the bonding properties of the long- and short-range states helps to explain the difference between the adsorption energies for DIB and MIB. The bonding in these two states can be mostly clearly seen by looking at the charge density difference. Figure $7(\mathrm{~b})$ and (c) show the charge density difference for the long- and short-range minima for DIB. The charge density difference is calculated by taking the charge density of the whole system and subtracting the charge densities of the isolated molecule and slab with the atoms fixed in the same positions as in the adsorbed state. The long-range structure shows bonding mainly between the iodine ions and the surface, whereas the short-range structure exhibits additional bonding between surface and the ring. Since there is an interaction via the iodine atoms MIB, with a single iodine atom, is less strongly bonded than DIB, with two iodine atoms, as seen by comparing Fig. 7(a) and (b).

To compare all adsorption geometries, the total charge displacement, denoted $Q_{\text {disp }}$, was calculated. $Q_{\text {disp }}$ is defined as follows

$$
Q_{\text {disp }}=\sum_{i}\left|Q_{\mathrm{tot}, i}-Q_{\mathrm{mol}, i}-Q_{\mathrm{srf}, i}\right|
$$

where $i$ is the grid index i.e. at each grid point the charge density for the molecule and slab (isolated but kept in their adsorption geometries) are subtracted from the charge density of the total system, and the absolute values are summed up over all grid points.

In general, $Q_{\text {disp }}$ increases as the number of iodine atoms increases, as shown in Fig. 8. However, for molecules with more than three iodine atoms the charge displacement depends strongly on the rotation of the molecule. The charge displacement and corresponding adsorption energies for each structure are presented in Table 2. In general, the higher the charge displacement, the stronger the adsorption. The correlation between charge displacement and adsorption energy is shown in Fig. 9. The lines show the 

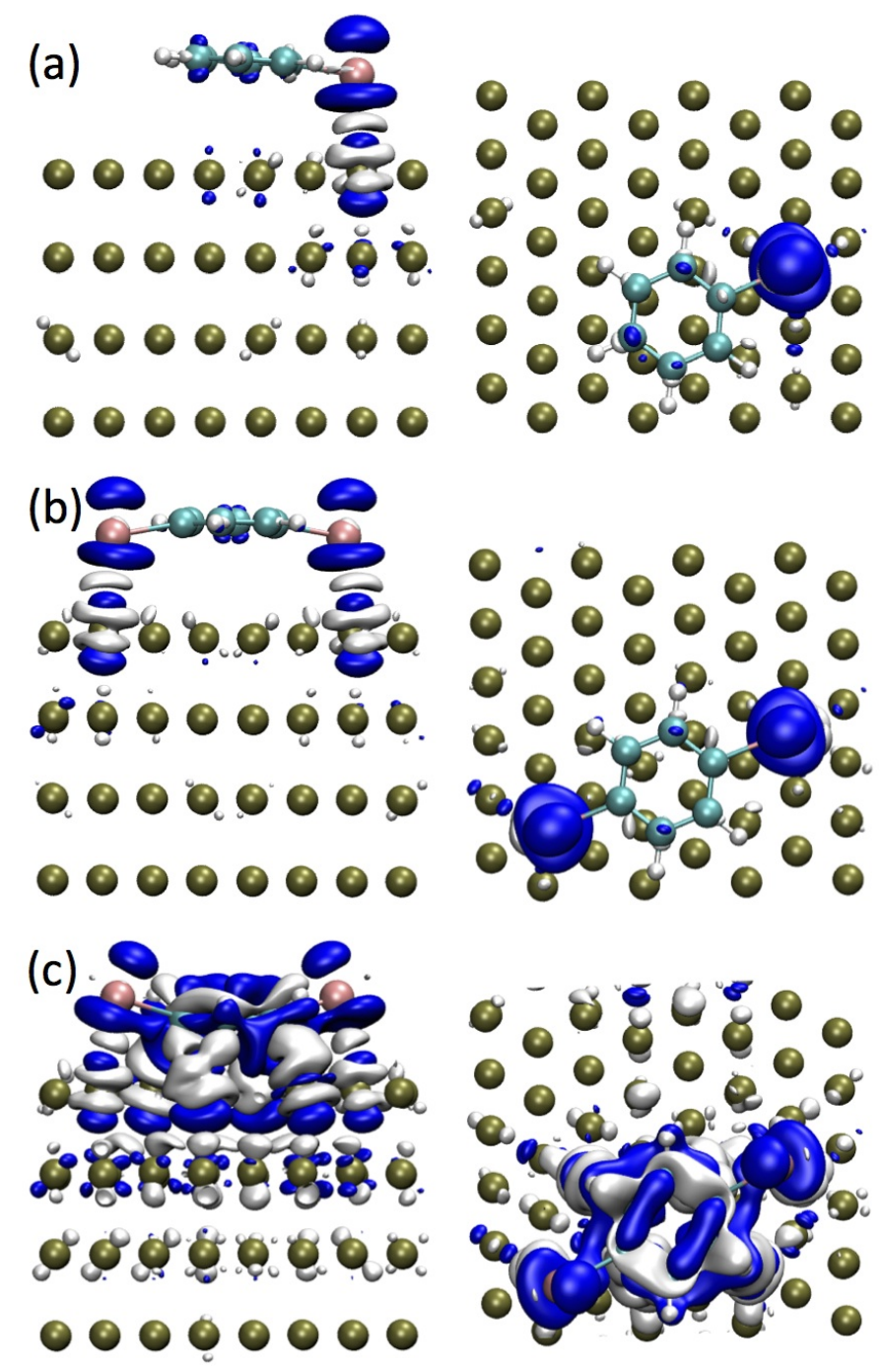

Figure 7: Side (left) and top (right) views of the isosurface of the charge density difference for (a) MIB in the long range structure, (b) DIB in the long range structure, and (c) DIB in the short range structure. Isosurfaces are shown for a charge of $\pm 3.6 \times 10^{-5} e / \AA^{3}$, where white is charge accumulation and blue is depletion. All charge densities shown are for $0.41 \mathrm{ML}$ coverage and are calculated using the vdW-DF functional. 


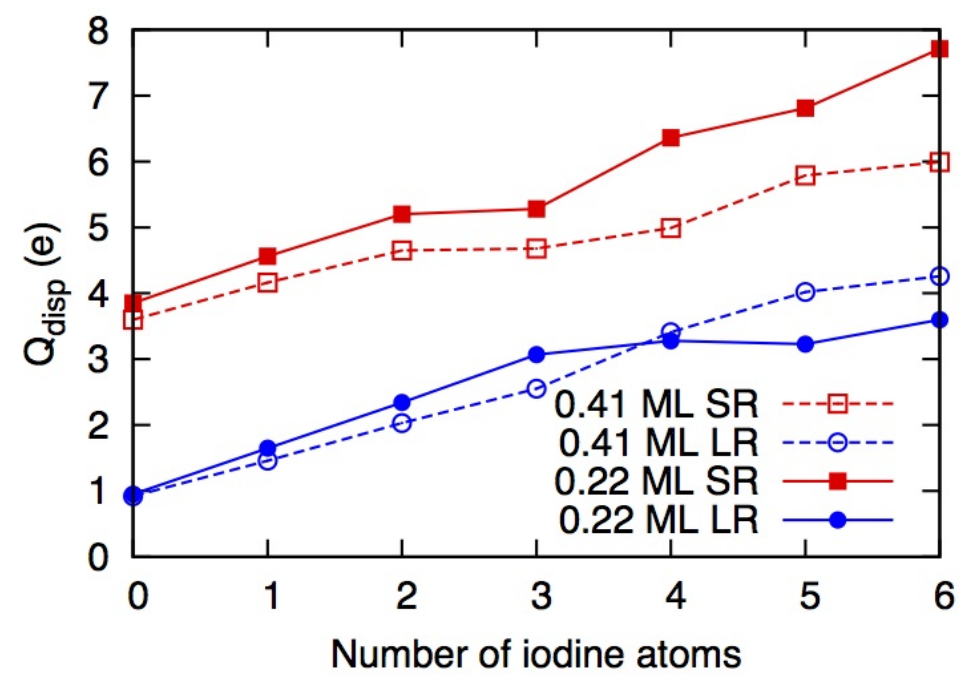

Figure 8: Variation of the difference in electronic charge distribution, $Q_{\text {disp }}$, with the number of iodine atoms. Only values for the most favorable rotations are shown.

best fits to the data with rms differences of $0.19,0.38,0.23$ and $0.43 \mathrm{eV}$ for the SR 0.22 ML, LR 0.22 ML, SR 0.41 ML and LR 0.41 ML structures, respectively. The change in coverage only makes a small difference to the correlation and in both cases the rms difference is smaller for the lower coverage of $0.22 \mathrm{ML}$. The SR structures exhibit a stronger correlation than the LR structures.

\subsection{Density of states}

The difference between the two states can also be understood by comparing the partial density of states (PDOS). The PDOS for the top Pt layer for both adsorption states is compared to that of the isolated (clean) surface, shown in Fig. 10(a). In the figure the PDOS have been aligned by their Fermi levels and the SR case has a slightly lower Fermi level by around $0.1 \mathrm{eV}$. As expected from the charge density differences, the SR adsorption state is more perturbed from the clean surface than the LR state.

The largest changes can be seen in the carbon and iodine $p$-states, shown in Fig. 10(b) and (c), respectively. To compare the adsorption states to the isolated (gas) molecule, the PDOS were aligned using the lowest band 


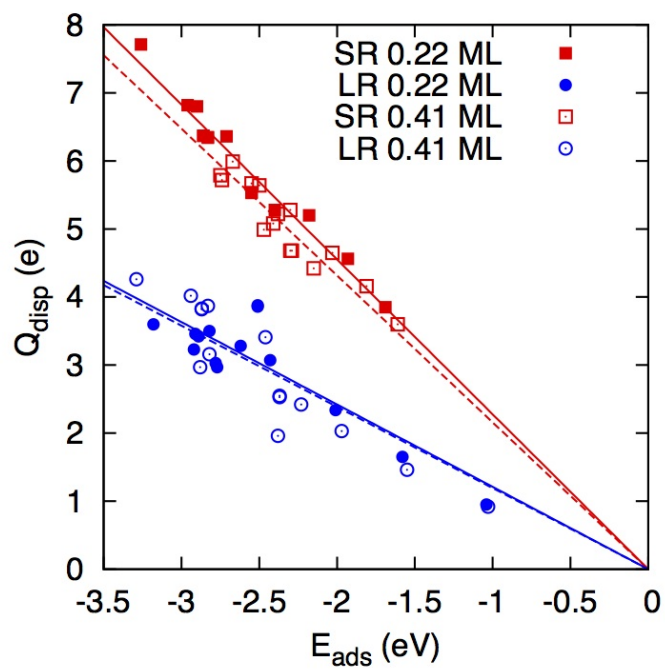

Figure 9: Correlation between $Q_{\text {disp }}$ and $E_{\text {ads }}$ for all short- and long-range structures at high and low coverage. In general for each adsorption structure at a particular coverage, the stronger the adsorption energy, the higher the total charge displacement.

energies. For carbon the LR state shares many of the same peaks as the isolated molecule but the SR state has very different features, with several large peaks either missing or shifted downwards in energy, notably the state at $\approx-2 \mathrm{eV}$. For both adsorption states the strong iodine peak at $\approx-6 \mathrm{eV}$ is significantly reduced and again the SR state shows a lowering in energy of the peak at $\approx-2$ e.

\subsection{Dissociation of monoiodobenzene}

According to experimental work, MIB dissociates at temperature at or above $175 \mathrm{~K}$ [15]. A dissociation mechanism was proposed, where the first step is $\mathrm{C}-\mathrm{I}$ bond scission resulting in the iodine atom adsorbed on the surface and the C-radical bonded to a surface Pt atom. We investigated the dissociated state and found that the ring was bonded via the $\mathrm{C}$ carbon atom to a $\mathrm{Pt}$ atom on the top layer of the $\mathrm{Pt}$ surface. The dissociated iodine atom was placed on an FCC site, which is known to be the preferred adsorption site [34]. The dissociated iodine atom on the FCC site is calculated to be 2.03-2.07 $\AA$ (depending on the site and ring orientation) (2.02 $\AA$ ) above the 


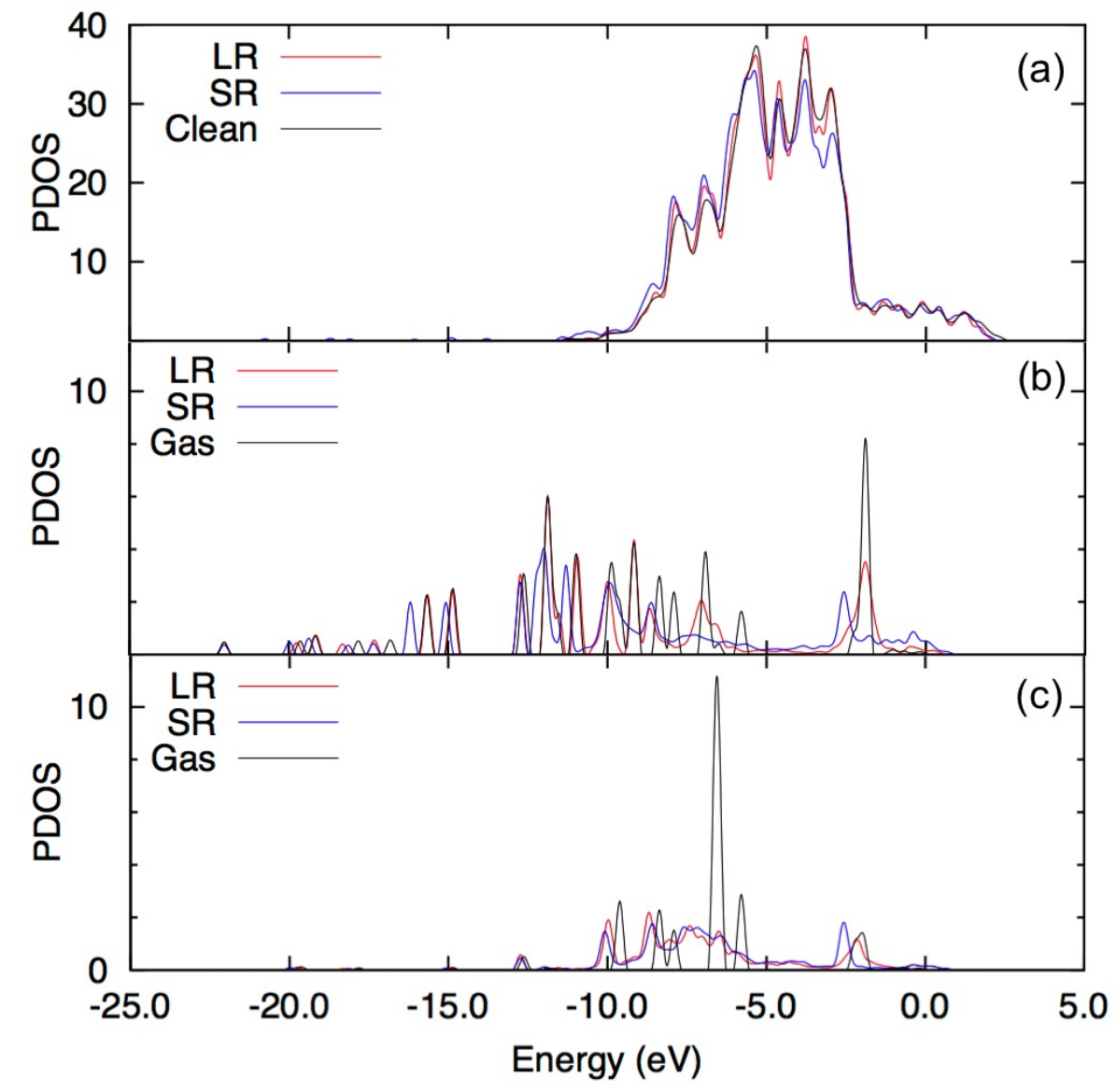

Figure 10: Partial density of states for LR and SR adsorption states for (a) top Pt layer, (b) carbon $p$-state and (c) iodine $p$-state. The Pt top layer PDOS was compared to the PDOS for the isolated slab and the PDOS in (a) are aligned by the Fermi levels. The C and I PDOS in (b) and (c) are compared to that of the isolated molecule and the PDOS are aligned using the lowest band energy.

surface, using the vdW-DF (PBE) functional. These values compare to a Hartree-Fock value of $2.9 \AA$ [34], an LDA value of $2.14 \AA$ [35] and a GGA value of $2.19 \AA$ [35]. The Pt-I bond distance from our calculation is $2.74 \AA$, which is smaller than the Hartree-Fock result of $3.0 \AA$.

First we investigate the structure of only the phenyl ring on the surface i.e. with the iodine atom removed from the surface. Two metastable struc- 
tures for the ring were found, as shown in Fig. 11. One $\mathrm{Pt}-\mathrm{C}$ bond forms an angle of $32.5^{\circ}$ with the surface and has a bond length of $2.03 \AA$. The $\mathrm{Pt}$ atom is raised from the surface by $0.23 \AA$. The other structure forms a higher $\mathrm{Pt}-\mathrm{C}$ angle of $74.6^{\circ}$ with the surface plane, has a bond length of $2.04 \AA$, and the $\mathrm{Pt}$ atom is raised from the surface by $0.25 \AA$. The lower angle of $32.5^{\circ}$ was found to be the most energetically favourable by $0.18 \mathrm{eV}$. To check if vdW forces were responsible for stabilising the flatter structure, it was also calculated with the PBE functional. The angle increased slightly to $36.4^{\circ}$ but it remained stable.
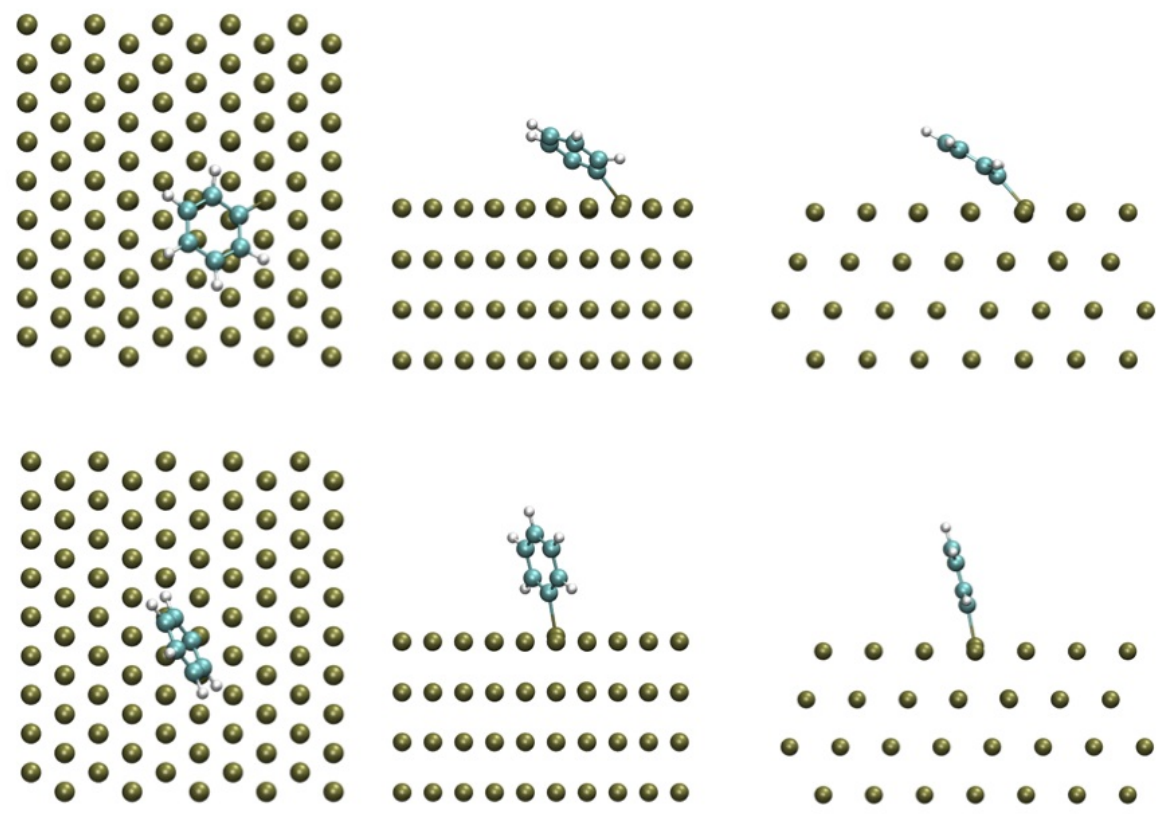

Figure 11: Adsorption geometries of dissociated phenyl ring with $0.22 \mathrm{ML}$ coverage. The top and bottom images show the ring with low and high angles, respectively.

This low angle is in agreement with a DFT-GGA study of phenyl on $\mathrm{Pt}(111)$ that found a tilt angle of $38^{\circ}$ [36], and also a DFT-GGA study of benzene dissociative adsorption on a bridge $30^{\circ}$ site, which found a tilt angle of $39.1^{\circ}$ [31]. However, the second study found that the angle increased to $56.6^{\circ}$ when the dissociated $\mathrm{H}$ atom was removed from the surface. To check the effect of the dissociated iodine atom on the ring, we also calcu- 
lated the adsorbed ring with the iodine placed on two different FCC hollow sites. The lowest energy structure for dissociated MIB using vdW-DF has the ring tilted at $32.8^{\circ}$ with respect to the surface and has an adsorption energy of $2.68 \mathrm{eV}$. The dissociated iodine atom, adsorbed on FCC sites has a negligible effect on the structure of the ring for both the high and lowtilted structures, although it reduces the difference in energies of the high and low tilted structures to $0.07 \mathrm{eV}$. This difference in energy is similar to the $10 \mathrm{~kJ} / \mathrm{mol}(\approx 0.10 \mathrm{eV})$ found in the first study mentioned above [36]. The DFT-GGA simulations of benzene adsorbed on the $\mathrm{Pt}(111)$ found that a tilted structure with angle $69.4^{\circ}$ was stable on the HCP0 site [31]. An experimental study of $\mathrm{C}-\mathrm{H}$ bending modes of $\mathrm{C}_{6} \mathrm{H}_{5}$ on $\mathrm{Pt}(111)$ estimated the tilt angle to be $73^{\circ}[15]$. NEXAFS experiments of bromobenzene adsorbed on $\mathrm{Pt}(111)$ observed intensities consistent with a ring tilt angle of $65^{\circ}$ [37]. Therefore, it is possible that several structures are present on the surface and experimental results may give an average over all these structures.

To understand the kinetics of the dissociation reaction, the energy barrier from the long-range state to the dissociated state was calculated using the elastic band method with 8 images and found to be $0.03 \mathrm{eV}$. This is smaller than the barrier from the long-range to short-range non-dissociated structure of $0.17 \mathrm{eV}$, shown in Fig. 1. This is consistent with the mechanism proposed by Cabibil in which iodobenzene initially physisorbs and then undergoes $\mathrm{C}-\mathrm{I}$ scission [15]. However, the barrier between the long- and short-range structure for MIB or DIB was not calculated using an NEB calculation and, thus, $0.17 \mathrm{eV}$ is an upper limit to the barrier. The barrier between the non-dissociated states is, therefore, not likely to be significantly higher than the dissociation barrier, and the short-range structure could occur. More experimental work is required to investigate the nature of the nondissociated adsorption structure.

\section{Conclusions}

The adsorption of iodobenzene molecules on the $\mathrm{Pt}(111)$ surface was investigated using density functional theory, including vdW forces. A bistability in non-dissociated adsorption was found for iodobenzene molecules on $\mathrm{Pt}(111)$. The long-range minimum is mainly due to the interaction between the iodine atoms and the surface, whereas the short-range minimum is mainly due to the interaction between the ring and the surface. For molecules with up to three iodine atoms at low coverage, the short-range adsorption structure is lower in energy than the long-range adsorption structure. As the num- 
ber of iodine atoms is increased to four or more, the short- and long-range structures have similar energy.

However, the ability to obtain the short-range states may be hampered by the tendency for iodobenzene to dissociate. The adsorption energies of the dissociated states of iodobenzene are lower in energy than the nondissociated states and for MIB the energy barrier between the long-range structure and the dissociated structure is smaller than the barrier from the long- to short-range structure.

This is a first investigation into the dissociation mechanism of iodobenzene on $\mathrm{Pt}(111)$ and further studies are required to investigate the full reaction pathways of polyiodobenzene molecules.

\section{Acknowledgements}

The authors gratefully acknowledge use of the Max Planck Institute's supercomputing facilities in Rechnungzentrum Garching. KJ was partially supported by the German Research Foundation's Priority Program SPP1369. RP and DD acknowledge funding from the MPRG program of the Max Planck Society.

\section{References}

[1] C. Joachim, J. K. Gimzewski, and A. Aviram. Electronics using hybridmolecular and mono-molecular devices. Nature, 408:541-548, 2000.

[2] Gregor Witte and Christof Wöll. Growth of aromatic molecules on solid substrates for applications in organic electronics. J. Mater. Res., 19:1889-1916, 2004.

[3] Johannes Barth, Giovanni Costantini, and Klaus Kern. Engineering atomic and molecular nanostructures at surfaces. Nature, 437:671-679, 2005.

[4] Takashi Yokoyama, Shiyoshi Yokoyama, Toshiya Kamikado, Yoshishige Okuno, and Shinro Mashiko. Selective assembly on a surface of supramolecular aggregates with controlled size and shape. Nature, 413:619-621, 2001.

[5] Marco Bieri, Manh-Thuong Nguyen, Oliver Gröning, Jinming Cai, Matthias Treier, Kamel Aït-Mansour, Pascal Ruffieux, Carlo A. 
Pignedoli, Daniele Passerone, Marcel Kastler, Klaus Müllen, and Roman Fasel. Two-Dimensional Polymer Formation on Surfaces: Insight into the Roles of Precursor Mobility and Reactivity. J. Am. Chem. Soc., 132:16669-16676, 2010.

[6] Wei Liu, Sergey N. Filimonov, Javier Carrasco, and Alexandre Tkatchenko. Molecular switches from Benzene Derivatives adsorbed on Metal Surfaces. Nat. Commun., 4:2569, 2013.

[7] Rengin Peköz, Karen Johnston, and Davide Donadio. Tuning the adsorption of aromatic molecules on Platinum via halogenation. J. Phys. Chem. C, 118:6235-6241, 2014.

[8] Haibao Huang, Ying Xu, Qiuyu Feng, and Dennis Y. C. Leung. Low temperature catalytic oxidation of volatile organic compounds: a review. Catal. Sci. Technol., 5:2649-2669, 2015.

[9] Norio Miyaura and Akira Suzuki. Previous Article Next Article Table of Contents Palladium-Catalyzed Cross-Coupling Reactions of Organoboron Compounds. Chem. Rev., 95:2457-2483, 1995.

[10] Shoji Matsumoto, Kenji Takase, and Katsuyuki Ogura. Novel Formation of Iodobenzene Derivatives from 1-(Methylthio)-3-tosylhexa1,3-dien-5-ynes via Iodine-Induced Intramolecular Cyclization. J. Org. Chem., 73:1726-1731, 2008.

[11] Vijay K. Kanuru, Georgios Kyriakou, Simon K. Beaumont, Anthoula C. Papageorgiou, David J. Watson, and Richard M. Lambert. Sonogashira Coupling on an Extended Gold Surface in Vacuo: Reaction of Phenylacetylene with Iodobenzene on $\mathrm{Au}(111)$. J. Am. Chem. Soc., 132:80818086, 2010.

[12] Denis Syomin and Bruce E. Koel. Adsorption of iodobenzene $\left(\mathrm{C}_{6} \mathrm{H}_{5} \mathrm{I}\right)$ on $\mathrm{Au}(111)$ surfaces and production of biphenyl $\left(\mathrm{C}_{6} \mathrm{H}_{5}\right)$. Surf. Sci., 490(3):265-273, 2001.

[13] Ming $\mathrm{Xi}$ and Brian E. Bent. Iodobenzene on $\mathrm{Cu}(111)$ : formation and coupling of adsorbed phenyl groups. Surf. Sci., 278:19-32, 1992.

[14] Karina Morgenstern, Saw Wai Hla, and Karl-Heinz Rieder. Coexisting superstructures of iodobenzene on $\mathrm{Cu}(111)$ near saturation coverage. Surf. Sci., 523:141-150, 2003. 
[15] H. Cabibil, H. Ihm, and J. M. White. The thermal chemistry of iodobenzene on Pt(111). Surf. Sci., 447:91-104, 2000.

[16] G. Kresse and J. Furthmüller. Efficiency of ab-initio total energy calculations for metals and semiconductors using a plane-wave basis set. Comput. Mat. Sci., 6:15, 1996.

[17] G. Kresse and J. Furthmüller. Efficient iterative schemes for ab initio total-energy calculations using a plane-wave basis set. Phys. Rev. B, 54:11169, 1996.

[18] P. E. Blöchl. Projector augmented-wave method. Phys. Rev. B, 50:17953, 1994.

[19] G. Kresse and D. Joubert. From ultrasoft pseudopotentials to the projector augmented-wave method. Phys. Rev. B, 59:1758, 1999.

[20] John. P. Perdew, Kieron Burke, and Matthias Ernzerhof. Generalized Gradient Approximation Made Simple. Phys. Rev. Lett., 77:3865, 1996.

[21] J.P. Perdew, K. Burke, and M. Ernzerhof. Erratum: Generalized Gradient Approximation Made Simple. Phys. Rev. Lett., 78:1396(E), 1997.

[22] M. Dion, H. Rydberg, E. Schröder, D. C. Langreth, and B. I. Lundqvist. Van der Waals Density Functional for General Geometries. Phys. Rev. Lett., 92:246401, 2004.

[23] Karen Johnston and Vagelis Harmandaris. Properties of Benzene Confined between Two $\mathrm{Au}(111)$ Surfaces Using a Combined Density Functional Theory and Classical Molecular Dynamics Approach. J. Phys. Chem. C, 115:14707, 2011.

[24] Karen Johnston, Jesper Kleis, Bengt I. Lundqvist, and Risto M. Nieminen. Influence of van der Waals Forces on the Adsorption Structure of Benzene on Silicon. Phys. Rev. B, 77:121404(R), 2008.

[25] R. W. G. Wyckoff. Crystal Structures. New York: Interscience, 2nd edition, 1963.

[26] Jiri Klimes, David R. Bowler, and Angelos Michaelides. Van der Waals density functionals applied to solids. Phys. Rev. B, 83:195131, 2011.

[27] Hyeran Ihm, Henry M. Ajo, J. M. Gottfried, P. Bera, and Charles T. Campbell. Calorimetric Measurement of the Heat of Adsorption of Benzene on Pt(111). J. Phys. Chem. B, 108:14627-14633, 2004. 
[28] G. Mills, H. Jonsson, and G. K. Schenter. Reversible work transition state theory: application to dissociative adsorption of hydrogen. Surf. Sci., 324:305, 1995.

[29] Hannes Jónsson, Greg Mills, and Karsten W. Jacobsen. Nudged elastic band method for finding minimum energy paths of transitions. World Scientific, 1998.

[30] C. Morin, D. Simon, and P. Sautet. Density-Functional Study of the Adsorption and Vibration Spectra of Benzene Molecules on Pt(111). J. Phys. Chem. B, 107:2995-3002, 2003.

[31] Weiwei Gao, Thomas A. Baker, Ling Zhou, Dilini S. Pinnaduwage, Efthimios Kaxiras, and Cynthia M. Friend. Chlorine Adsorption on $\mathrm{Au}(111)$ : Chlorine Overlayer or Surface Chloride? J. Am. Chem. Soc., 130:3560-3565, 2008.

[32] R. Peköz, K. Johnston, and D. Donadio. Adsorption of dichlorobenzene on $\mathrm{Au}$ and $\mathrm{Pt}$ stepped surfaces using van der Waals density functional theory. J. Phys. Chem. C, 116:20409, 2012.

[33] Wei Liu, Victor G Ruiz, Guo-Zu Zhang, Biswajit Santra, Xinguo Ren, Matthias Scheffler, and Alexandre Tkatchenko. Structure and energetics of benzene adsorbed on transition-metal surfaces: density-functional theory with van der Waals interactions including collective substrate response. New J. Phys., 15:053046, 2013.

[34] Paul S. Bagus, Christof Wöll, and Andrzej Wieckowski. Dependence of surface properties on adsorbate-substrate distance: Work function changes and binding energy shifts for I/Pt(111). Surf. Sci., 603:273$283,2009$.

[35] Alexandre Tkatchenko, Nikola Batina, Andres Cedillo, and Marcelo Galvan. Charge transfer and adsorption energies in the iodine-Pt(111) interaction. Surf. Sci., 581:58-65, 2005.

[36] Mark Saeys, Marie-Francoise Reyniers, Matthew Neurock, and Guy B. Marin. Density Functional Theory Analysys of Benzene (De)Hydrogenation on $\mathrm{Pt}(111)$ : Addition and Removal of the First Two H-Atoms. J. Phys. Chem. B, 107:3844-3855, 2003.

[37] Adam F. Lee, Zhipeng Chang, Simon F. J. Hackett, Andrew D. Newman, and Karen Wilson. Hydrodebromination of bromobenzene over Pt(111). J. Phys. Chem. C, 111:10455-10460, 2007. 